\title{
Individual alpha peak frequency is related to latent factors of general cognitive abilities
}

\author{
Thomas H. Grandy ${ }^{\text {a }}$, Markus Werkle-Bergner ${ }^{\mathrm{a}, *}$, Christian Chicherio ${ }^{\mathrm{a}, \mathrm{b}}$, Martin Lövdén ${ }^{\mathrm{a}, \mathrm{c}}$, \\ Florian Schmiedek $^{\mathrm{a}, \mathrm{d}}$, Ulman Lindenberger ${ }^{\mathrm{a}}$ \\ a Center for Lifespan Psychology, Max Planck Institute for Human Development, Berlin, Germany \\ b Neuropsychology Unit, Neurology Clinic, Department of Clinical Neurosciences, Geneva University Hospitals, Switzerland

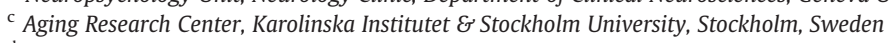 \\ d German Institute for International Educational Research (DIPF), Frankfurt am Main, Germany
}

\section{A R T I C L E I N F O}

\section{Article history:}

Accepted 15 April 2013

Available online 25 April 2013

\section{Keywords:}

Alpha frequency

Neural oscillations

EEG

General intelligence

\begin{abstract}
A B S T R A C T
Some eighty years after the discovery of the human electroencephalogram (EEG) and its dominant rhythm, alpha $(\sim 10 \mathrm{~Hz})$, the neurophysiological functions and behavioral correlates of alpha oscillations are still under debate. Similarly, the biological mechanisms contributing to the general factor of intelligence, or $g$, have been under scrutiny for decades. Individual alpha frequency (IAF), a trait-like parameter of the EEG, has been found to correlate with individual differences in cognitive performance and cognitive abilities. Informed by large-scale theories of neural organization emphasizing the general functional significance of oscillatory activity, the present study replicates and extends these findings by testing the hypothesis that IAF is related to intelligence at the level of $g$, rather than at the level of specific cognitive abilities. Structural equation modeling allowed us to statistically control for measurement error when estimating the association between IAF and intellectual functioning. In line with our hypothesis, we found a statistically reliable and substantial correlation between IAF and $g(r=.40)$. The magnitude of this correlation did not differ significantly between younger and older adults, and captured all of the covariation between IAF and the cognitive abilities of reasoning, memory, and perceptual speed. The observed association between IAF and $g$ provides a parsimonious explanation for the commonly observed diffuse pattern of correlations between IAF and cognitive performance. We conclude that IAF is a marker of global architectural and functional properties of the human brain.
\end{abstract}

(c) 2013 Elsevier Inc. All rights reserved.

\section{Introduction}

Understanding the mapping of anatomical and functional properties of the brain onto individual differences in cognitive functioning is a major goal of cognitive neuroscience and central to attempts at delineating the neural mechanisms associated with intelligent behavior (Deary et al., 2010; Jung and Haier, 2007; Kanai and Rees, 2011; Narr et al., 2007; Toga and Thompson, 2005). The notion that characteristics of the human electroencephalogram (EEG) are related to measures of intelligence has a long history going back almost as far as the discovery of the EEG by Hans Berger (1929, 1930), with the alpha rhythm being one of the first targets for studying the relationship between EEG markers and intelligence. It is easily discerned in the raw EEG traces at parieto-occipital electrodes when persons are awake and relaxed with their eyes closed (Adrian and Matthews, 1934; Adrian and Yamagiwa, 1935). The average of alpha frequency

\footnotetext{
* Corresponding author at: Center for Lifespan Psychology, Max Planck Institute for Human Development, Lentzeallee 94, 14195 Berlin, Germany.

E-mail address: werkle@mpib-berlin.mpg.de (M. Werkle-Bergner).
}

is around $10 \mathrm{~Hz}$, with a range of approximately 8-12 $\mathrm{Hz}$ in healthy adults (cf. Aurlien et al., 2004; Chiang et al., 2011; Niedermeyer and Lopes da Silva, 1999). One of the earliest observations relating the alpha frequency (AF) to individual differences in cognitive functioning was the finding of 'mentally retarded' patients (Berger, 1933) exhibiting systematically slower alpha waves. Since then it has remained an appealing notion that 'smarter brains are running faster' (Posthuma et al., 2001), with a long history of findings supporting this idea but also with inconsistent reports (see below).

From a psychometric perspective, AF is a promising neurophysiological trait marker for investigating the association between brain and cognitive functioning (Grandy et al., in press). The AF is characterized by a remarkably high relative and mean test-retest stability in samples of healthy individuals (Deakin and Exley, 1979; Gasser et al., 1985; Kondacs and Szabó, 1999; Salinsky et al., 1991), with test-retest intervals ranging up to several years (Kondacs and Szabó, 1999). Stability coefficients of AF derived from EEG recordings with closed eyes were found to be typically around .75 to .90 , with higher correlations for shorter test-retest intervals. It follows that $\mathrm{AF}$ shows trait-like characteristics, with substantial and stable differences between individuals that justify use of the term, individual alpha 
frequency (IAF), to denote the AF of a given person (cf. Doppelmayr et al., 1998; Klimesch, 1996, 1997). In full agreement with this line of reasoning, IAF has been found to show considerable heritability (Lykken et al., 1974; Posthuma et al., 2001; Smit et al., 2006; van Baal et al., 2001; van Beijsterveldt and Boomsma, 1994; van Beijsterveldt and van Baal, 2002; Vogel, 1970).

The magnitude and consistency of the association between IAF and individual differences in intellectual functioning are a matter of ongoing debate (cf. Anokhin and Vogel, 1996; Ellingson, 1966; Posthuma et al., 2001; Vogel and Broverman, 1964). Several studies have reported significant correlations between IAF and verbal abilities (Angelakis et al., 2004a; Anokhin and Vogel, 1996; Mundy-Castle, 1958), memory performance (Klimesch et al., 1990, 1993; Lebedev, 1994; Saletu and Grunberger, 1985), Digit Span performance (Angelakis et al., 2004b; Clark et al., 2004), performance on the Raven matrices (Anokhin and Vogel, 1996), response control (Angelakis et al., 2004a), speed of information processing (Klimesch et al., 1996; Mundy-Castle, 1958), reaction times (Surwillo, 1961, 1963, 1964), and indicators of general intelligence (Giannitrapani, 1985; Mundy-Castle, 1958; Mundy-Castle and Nelson, 1960). Collectively, these reports indicate that IAF is functionally related to various types of cognitive performance. At the same time, some studies failed to detect any associations between IAF and cognitive performance (Ellingson, 1966; Posthuma et al., 2001; Vogel and Broverman, 1964). Moreover, some of the initial findings, such as correlations between IAF and verbal subtests of the Wechsler Intelligence Test (Angelakis et al., 2004a; Mundy-Castle, 1958), were not replicated in later work (Posthuma et al., 2001). However, it needs to be cautioned that many of the studies had small sample sizes. In addition, associations between IAF and intelligence were often computed at the level of individual cognitive tests. Generally, such tests are poor indicators of intelligence because their reliability and validity is far from perfect. In sum, it is conceivable that combinations of sampling error, lack of statistical power, measurement error as well as variance specific to individual cognitive tests have obscured, and potentially lowered, the observable association between IAF and intelligence.

Despite these limitations, the predominantly positive correlations between IAF and a variety of cognitive performance measures point to an association between IAF and the positively correlated manifold of intellectual abilities (cf. Deary et al., 2010). Given its high stability and heritability, it is plausible to assume that IAF is linked to cognitive performance in a general rather than in a specific manner. Specifically, we would like to propose that IAF is a physiological marker of Spearman's 'general intelligence' (Spearman, 1904). It has been shown that secondorder factors extracted from a wide range of intelligence test batteries tend to be highly correlated (Johnson et al., 2004, 2008), suggesting that the common variance extracted from different intelligence test batteries can be seen as different expressions of a common construct termed general intelligence, or $g$. Hence, we predicted that allowing for an association between IAF and intelligence at the level of $g$ would obliterate the need to model the IAF-intelligence link at lower levels of the intelligence hierarchy.

We tested these predictions using data from the COGITO study (Schmiedek et al., 2010). The COGITO study was conducted to investigate day-to-day variability of cognitive performance and effects of extensive cognitive training. Within the COGITO study, a large sample of 145 younger adults (20-31 years) and 142 older adults (65-81 years) completed 27 subtests of the Berlin Intelligence Structure test (BIS; Jäger et al., 1997) from the cognitive domains perceptual speed, memory, and reasoning as part of a large battery of baseline assessments. Additionally, approximately $30 \%$ of the participants - 45 younger and 40 older adults - took part in two EEG sessions, one directly after the baseline assessments and the other on average 6.6 months later. Resting state recordings with eyes closed and eyes open were obtained at each of these two occasions.

The constructs in the BIS test broadly cover the range and type of cognitive tests that have been shown to correlate with IAF in previous work (see above). Importantly, by making use of the fairly large full COGITO parent sample $(n=287)$, we were able to establish a sound confirmatory factor model representing the structure of intelligence by three first-order factors and one second-order latent factor $g$. Following the confirmation of this factor structure, structural equation modeling (SEM) with full information maximum likelihood (FIML) estimation was used to project a latent IAF factor, which was derived from eyes closed and eyes open resting state EEG measurements, into the latent space of intellectual abilities. This procedure allowed us to estimate the association between IAF and $g$ unbiased by measurement error at the latent level, and to test whether links of IAF to cognitive performance at lower levels of the hierarchy (i.e., first-order factors, specific tests) are needed after accounting for the link between IAF and $g$. If there were no need to estimate such residual associations, this would be consistent with the guiding hypothesis that IAF-intelligence associations reflect general properties of the human brain. To our knowledge, the association between IAF and intelligence has not been investigated using SEM techniques.

\section{Methods}

\section{Participants}

The full sample of the COGITO study involved 145 younger adults (YA; 72 women, $M_{\text {age }}=25.5, S D=2.7$, range $=20-31$ years; intervention group $n=101$, control group $n=44$ ) and 142 older adults $\left(\mathrm{OA} ; 70\right.$ women; $M_{\text {age }}=71.1 ; S D=4.0$; range $=65-81$ years; intervention group $n=103$, control group $n=39$ ); for details, see Schmiedek et al. (2010). The participants were recruited through newspaper advertisements, word-of-mouth recommendation, and flyers circulated in Berlin. Intervention and control groups were matched on age and cognitive status (see Schmiedek et al., 2010).

Table 1

Sample descriptives.

\begin{tabular}{|c|c|c|c|c|}
\hline & \multicolumn{2}{|l|}{ Younger adults } & \multicolumn{2}{|l|}{ Older adults } \\
\hline & $\begin{array}{l}\text { COGITO sample } \\
(n=145)\end{array}$ & $\begin{array}{l}\text { EEG sub-sample } \\
(n=45)\end{array}$ & $\begin{array}{l}\text { COGITO sample } \\
(n=142)\end{array}$ & $\begin{array}{l}\text { EEG sub-sample } \\
(n=40)\end{array}$ \\
\hline Age & $25.5(2.7)$ & $25.1(2.8)$ & $71.1(4.0)$ & $70.3(3.9)$ \\
\hline Digit symbol & $60.1(9.3)$ & $60.4(8.9)$ & $43.9(8.8)$ & $45.8(7.6)$ \\
\hline Spot-a-Word & $.66(.11)$ & $.67(.10)$ & $.80(.10)$ & $.83(.08)$ \\
\hline Raven matrices & $.52(.20)$ & $.54(.19)$ & $.25(.15)$ & $.29(.16)$ \\
\hline MMSE & - & - & $28.3(1.3)$ & $28.5(1.4)$ \\
\hline IAF EC & - & $9.8(0.8)$ & - & $9.3(0.8)$ \\
\hline IAF EO & - & $10.0(0.9)$ & - & $9.4(1.2)$ \\
\hline
\end{tabular}

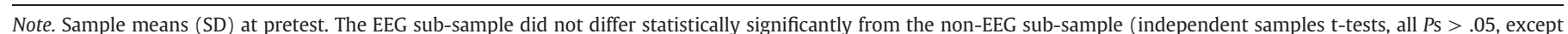
Spot-a-Word accuracy in the older adults, $P<.05)$. MMSE $=$ Mini-mental state examination; $\mathrm{IAF}=$ individual alpha frequency; $\mathrm{EC}=$ eyes closed; EO $=$ eyes open. 
Approximately $30 \%$ of the parent sample participated in two EEG recordings conducted in the context of pre- and post-test assessments, respectively, resulting in an effective EEG sub-sample of 45 younger adults (24 women; $M_{\text {age }}=25.1 ; S D=2.8$; range $=20-31$ years) and 40 older adults (OA; 16 women; $M_{\text {age }}=70.3 ; S D=3.9$; range $=65-80$ years). The EEG and non-EEG subsamples were comparable on age and cognitive status (see Table 1; independent samples t-tests, all Ps $>.05$, except Spot-a-Word accuracy in the older adults, $P<.05)$. All the participants in the EEG sample were right-handed, had normal or corrected-to-normal vision, and reported no history of cardiovascular disease (except treated hypertension), diabetes, neurological or psychiatric conditions, or drug/alcohol abuse. No use of neuropharmacologically acting drugs like anti-epileptic or anti-depressant drugs was reported. Older participants were screened for dementia using the mini-mental state examination (MMSE; Folstein et al., 1975) with a cut-off score of 26 for the EEG sub-sample. The ethics committee of the MPIB approved of the study, and all the participants provided written informed consent.

\section{Study design}

The overall design of the COGITO study consisted of an initial extensive assessment (pretest; ten sessions à 2-2.5 h) of a wide range of cognitive tests and self-report questionnaires (e.g., personality inventories), followed by a longitudinal intervention phase of on average 101 one-hour cognitive training sessions (intervention group) or an equal time interval with no contact (control group), and a final extensive assessment identical to the pretest (posttest). During the intervention phase, six perceptual speed tasks, three working memory tasks and three episodic memory tasks were administered in every session (see Schmiedek et al., 2010, for a detailed description of the study design and cognitive tasks). For the EEG sub-sample, EEG recordings were acquired at pre- and post-tests shortly after the ten sessions of behavioral pre- and post-tests had been completed.

The Berlin Intelligence Structure (BIS) test data reported here were acquired in one of the pretest sessions. The BIS was administered before the intervention, and experimental and control groups have been shown not to differ reliably in relation to age and cognitive status (Schmiedek et al., 2010). Therefore, data from intervention and control groups were pooled in the analyses reported here.

\section{Berlin Intelligence Structure (BIS-4) battery}

The Berlin Intelligence Structure test (BIS; Jäger et al., 1997) has been constructed on the basis of a hierarchical model of intelligence distinguishing between content and operation facets (cf. Wilhelm and Schulze, 2002; p. 543). The content facet refers to the 'material' cognition operates on, and differentiates between verbal, numerical, and figural contents, whereas the operation facet discriminates between perceptual speed, memory, reasoning, and creativity. In the COGITO study, the perceptual speed, memory, and reasoning facets of the BIS test were assessed, with each of the facets being represented by nine tasks each, namely, three from each content domain. The tasks are briefly described in the following sections.

\section{BIS perceptual speed tasks}

TG (Part-Whole): In a list of 28 words, all words for which the current word denoted a part of the previous word (e.g., "year"-"month") had to be marked with a cross as quickly as possible. UW (Word completion): In a list of 57 nouns, one missing letter for each noun had to be filled in as quickly as possible. KW (Plants): In a list of 100 words, all the words naming a plant had to be marked as quickly as possible. SI (Division by seven): In a list of 91 numbers, the numbers that can be divided exactly by seven had to be marked as quickly as possible. $X G$ (X-Greater): Participants were given a list of 130 numbers and told to mark those that are 3 integers greater than the preceding number as quickly as possible. $R Z$ (Arithmetic operator): In a list of 20 equations of the kind "2_3_1 = 4", participants had to enter "plus" or "minus" signs as quickly as possible between the numbers to produce a correct equation. OE (Old English): In rows of lower case letters, participants had to mark those printed in a gothic font as quickly as possible. ZS (Digit symbol): Maximally 68 symbols had to be drawn below strings of single digits (1-9) according to a digit-symbol key provided. BD (Findings $x \mathrm{~s}$ ): All the $x \mathrm{~s}$ in a row of lower case letters had to be marked as quickly as possible.

\section{BIS memory tasks}

WM (Word memory): Recall of a list of 20 nouns. PS (Fantasy language): 20 pairs of German words and words of a nonsense language had to be memorized. Immediately afterwards the correct paired nonsense words for given German words had to be recognized out of 5 alternatives. ST (Story recall): Recall of several facts of a short story. ZZ (Number memory): Recall of a list of 16 2-digit numbers. ZP (Number pair memory): Cued recall of 12 number pairs; cues were 2 digits long, numbers to recall 3 digits long. ZW (Number recognition): Recognition of a list of nine 5-digit numbers out of nine times six 5-digit numbers. FM (Company logos): A set of 20 company logos with pictorial figures on different geometric shapes had to be memorized within $60 \mathrm{~s}$. In the recall phase, the correct corresponding shapes for each figure had to be correctly identified from 4 shapes shown. WE (Route memory): On a stylized map with shaded areas denoting blocks of buildings, the marked route from one place to another had to be memorized within $30 \mathrm{~s}$ and later reproduced on a copy of the map. OG (Location memory): On a town map, 27 buildings were colored in black. After $90 \mathrm{~s}$ for memorization, the buildings had to be marked on a copy of the map.

\section{BIS reasoning tasks}

WS (Odd-one-out; 9 items): One of four words in a line differed in meaning from the other words. This had to be crossed out by participants. SL (Formal-logic reasoning; $3 \times 2$ statements and 16 inferences total): Participants were given statements and had to evaluate whether inferences following from these statements were logically valid or not. WA (Verbal analogies; 8 items): Participants had to chose one out of five alternatives to complete a verbal analogy of the type A: $\mathrm{B}=\mathrm{C}:$ ? ZN (Number series; 9 items): Participants had to complete sequences of numbers based on certain rules. TL (Climate information table; 6 items): Participants had to answer multiple-choice questions based on data provided in a table of summary statistics on temperatures and rainfall measures. SC (Arithmetic; 7 items): Participants had to correctly identify the solutions to equations involving large numbers, identifying one out of 5 possible solutions. Exact arithmetic calculation was not needed, as the correct answer could be identified by applying general arithmetic reasoning. AW (Surface development; 5 items): Participants were given an unfolded representation of a geometrical shape. They then had to indicate the shape into which this unfolded representation can be folded by selecting from five given bodies that varied in form and perspective. FA (Tangram; 6 items): Participants were given parts of a geometrical figure and had to indicate which figure out of five alternatives could be assembled with them. CH (Figural series; 6 items): Participants had to add the next two members of a sequence of line drawings composed according to certain rules.

Content facets were represented by following sub-tests, for perceptual speed: TG, KW, UW (verbal), XG, SI, RZ (numerical), and $\mathrm{BD}, \mathrm{OE}, \mathrm{ZS}$ (figural); for memory: ST, WM, PS (verbal) ZP, ZZ, ZW (numerical), and OG, FM WE (figural); for reasoning: WA, WS, SL (verbal), $\mathrm{ZN}, \mathrm{SC}, \mathrm{TL}$ (numerical), and $\mathrm{CH}, \mathrm{FA}, \mathrm{AW}$ (figural). For each operation facet, the nine sub-tests were $z$-standardized within age groups and aggregated into three parcels (see Table 2 for inter-correlations) serving as indicator variables for the latent factors perceptual speed, memory and reasoning, respectively (cf. Schmiedek et al., 2009; Wilhelm and Schulze, 2002). 
Table 2

Positive manifold of aggregated cognitive scores (parcels) from the BIS test.

\begin{tabular}{|c|c|c|c|c|c|c|c|c|}
\hline & $\begin{array}{l}\text { Speed }_{1} \\
\text { XG/OE/KW }\end{array}$ & $\begin{array}{l}\text { Speed }_{2} \\
\text { SI/ZS/UW }\end{array}$ & $\begin{array}{l}\text { Speed }_{3} \\
\text { RZ/BD/TG }\end{array}$ & $\begin{array}{l}\text { Memory }_{1} \\
\text { ZP/ST/OG }\end{array}$ & $\begin{array}{l}\text { Memory }_{2} \\
\text { ZZ/WM/FM }\end{array}$ & $\begin{array}{l}\text { Memory }_{3} \\
\mathrm{ZW} / \mathrm{PS} / \mathrm{WE}\end{array}$ & $\begin{array}{l}\text { Reasoning }_{1} \\
\text { FA/ZN/WA }\end{array}$ & $\begin{array}{l}\text { Reasoning }_{2} \\
\mathrm{CH} / \mathrm{TL} / \mathrm{WS}\end{array}$ \\
\hline \multicolumn{9}{|l|}{ Speed $_{1}$} \\
\hline Speed $_{2}$ & $.66 / .66$ & & & & & & & \\
\hline Speed $_{3}$ & $.68 / .69$ & $.55 / .62$ & & & & & & \\
\hline Memory $_{1}$ & $.29 / .48$ & $.48 / .41$ & $.26 / .45$ & & & & & \\
\hline Memory $_{2}$ & $.28 / .40$ & $.44 / .33$ & $.22 / .34$ & .62 /.62 & & & & \\
\hline Memory $_{3}$ & $.30 / .28$ & $.38 / .32$ & $.31 / .25$ & $.60 / .41$ & $.54 / .37$ & & & \\
\hline Reasoning $_{1}$ & $.35 / .35$ & $.47 / .22$ & $.40 / .31$ & $.37 / .28$ & $.39 / .25$ & $.42 / .18^{*}$ & & \\
\hline Reasoning $_{2}$ & $.44 / .38$ & $.48 / .37$ & $.43 / .39$ & $.40 / .34$ & $.27 / .23$ & $.32 / .36$ & .67 / .54 & \\
\hline Reasoning $_{3}$ & $.34 / .32$ & $.38 / .30$ & $.40 / .41$ & $.34 / .23$ & $.25 / .22$ & .39 / .22 & $.68 / .39$ & $.57 / .50$ \\
\hline
\end{tabular}

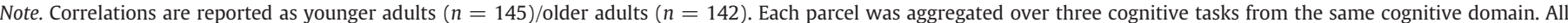
correlations are highly significant $[P<.01]$, except ${ }^{*}[P<.05]$.

\section{EEG data acquisition}

EEG was recorded continuously with BrainAmp amplifiers (Brain Products $\mathrm{GmbH}$, Gilching, Germany) from $64 \mathrm{Ag} / \mathrm{AgCl}$ electrodes. Sixty scalp electrodes, embedded in an elastic cap (EASYCAP GmbH, Herrsching, Germany), were organized according to the $10 \%$ system (cf. Oostenveld and Praamstra, 2001). Ground was placed at AFz. Two electrodes were placed on the outer canthi (horizontal EOG) and one electrode below the left eye (vertical EOG) in order to monitor eye movements. During recording, all electrodes were referenced to the right mastoid electrode, while the left mastoid electrode was recorded as an additional channel. Electrode impedances were maintained below $5 \mathrm{k} \Omega$ before recording. The EEG was recorded with an analog pass-band of 0.1 to $250 \mathrm{~Hz}$ and digitized with a sampling rate of $1 \mathrm{kHz}$. Resting state EEG data included in this study stem from the two EEG recording sessions of each participant (preand post-tests). Resting state EEG was assessed from two conditions in each session: 2 min of recording with eyes closed (EC) and 2 min of recording with eyes open (EO). The participants were instructed to sit as relaxed and still as possible and to fixate a fixation cross during the eyes open condition.

\section{EEG data preprocessing}

Preprocessing and analysis of EEG data were performed using the EEGLAB (Delorme and Makeig, 2004) and Fieldtrip toolboxes (Oostenveld et al., 2011) as well as custom-written MATLAB (The MathWorks Inc., Natick, MA, USA) code. EEG data were re-referenced to mathematically linked mastoids, filtered with a 4th order Butterworth filter and a pass-band of 0.5 to $100 \mathrm{~Hz}$, and segmented into epochs of $1.024 \mathrm{~s}$. Segments were visually inspected and all segments containing artifacts other than eye blinks and eye movements were excluded from further analyses. After manual artifact rejection an independent component analysis (ICA; Bell and Sejnowski, 1995) was conducted to correct for eye blinks and movements. Artifact corrected data were then subjected to a fast Fourier transformation (FFT) within the Fieldtrip toolbox, using a Hanning-window and zero-padding to $10 \mathrm{~s}$ to obtain a frequency resolution of $0.1 \mathrm{~Hz}$.

\section{Estimation of individual alpha frequency (IAF)}

Individual alpha frequency (IAF) was estimated as peak alpha frequency from the mean spectrum over 17 posterior electrodes (Pz, P1/2, P3/4, P5/6, P7/8, POz, PO3/4, PO7/8, Oz and 01/2) by means of peak detection between 7.5 and $12.5 \mathrm{~Hz}$. IAF was estimated separately for the EC and EO conditions. We have previously shown for this dataset (Grandy et al., in press) that reliable IAF values can be obtained for both conditions and that both conditions share a considerable amount of variance (see also Table 3). Peaks were defined as those frequency points where the first derivative of the spectrum changed from positive to negative. Searching explicitly for peaks assured that we extracted true peaks from the spectrum rather than arbitrary maximal values at the boundary of the predefined alpha range. Only in one out of 340 peak estimations (0.3\%) no peak was found in the alpha range.

A common problem in determining IAF is the existence of two peaks within the alpha-frequency range (cf. Posthuma et al., 2001). For the present dataset, double peaks in the alpha range were observed in 12 participants (14.1\%) with eyes closed and in 20 participants (23.5\%) with eyes open. However, in only three participants (3.5\%) with eyes closed and seven participants (8.2\%) with eyes open, differing maximum peaks were found across conditions. Since, as described below, we calculated the mean IAF across two sessions and estimated the relationship between IAF and intelligence at the latent level, we did not expect these cases to have a large impact on the results. Control analyses (not reported here), with exclusion of data points from conditions with inconsistent double peaks as well as exclusion of all conditions where double peaks were observed, did not change the results, underscoring the robustness of our reported findings.

For every participant two IAF values were determined. In previous work focusing on the stability of IAF within the COGITO study (Grandy et al., in press), we have shown that IAF was not affected by extensive cognitive training: repeated measures ANOVA with factors time (pretest vs. posttest) $\times$ group (intervention vs. control) $\times$ condition (eyes closed vs. eyes open) $\times$ age (younger adults vs. older adults) did not show any significant main effects or interactions (all $F s(1,80)<2.97$; Ps $>.05$ ), except for the factor age $(F(1,80)=5.07, P<.05)$, indicating slower IAF in

Table 3

Correlation between IAF and aggregated cognitive ability scores.

\begin{tabular}{lcc}
\hline & EC & EO \\
\hline EO & $.87^{* *} / .52^{* *}$ & \\
Speed & $.31^{*} / .06$ & $.34^{*} / .15$ \\
Memory & $.37^{*} / .36^{*}$ & $.23 / .34^{*}$ \\
Reasoning & $.25 / .06$ & $.21 / .06$ \\
\hline
\end{tabular}

Note. Correlations are reported as younger adults $(n=45)$ /older adults $(n=40)$. Aggregated scores were calculated by taking the mean over parcels. IAF $=$ individual alpha frequency; $\mathrm{EC}=$ eyes closed; $\mathrm{EO}=$ eyes open. ${ }^{*}: P<.05 ;{ }^{* *}: P<.01$. 
older adults on average. Therefore, we aggregated IAF values from preand post-tests separately for the EC and EO conditions.

\section{Data analysis}

Sample descriptives were computed using SPSS 15.0 (SPSS Inc., Chicago, IL, USA). Structural equation modeling (SEM), including confirmatory factor analysis (CFA), was conducted with Mplus (Version 6.1, Muthén and Muthén, 1998-2010). SEM is a multivariate statistical tool, nowadays routinely used in the behavioral and social sciences (Bollen, 1989; Kline, 2005) and increasingly gaining attention in the neurosciences (e.g., Lövdén et al., 2013; Raz et al., 2005; Ziegler et al., 2012). It allows testing structural hypotheses about associations and influences among multiple variables by examining how well a given model is able to reproduce the variance-covariance matrix of a set of observed variables. A SEM model typically consists of a measurement model, specifying the relationship of a number of observed variables to latent (unobserved) variables (cf. Bollen, 2002). Latent variables are comparable to factors in factor analysis, that is, they are not measured but rather represent the variance shared by the observed (measured) variables. In our case, the latent variables are the specific ability factors perceptual speed, memory, reasoning, as well as IAF, each represented by two to three observed variables or aggregates of observed variables, respectively. The latent variables can be assumed to be free of task-specific sources of variance as well as measurement error. The core of a SEM is the relationship between latent variables-here, how individual differences of perceptual speed, memory, and reasoning can be accounted for by a second order factor $g$, and the relationship between $g$ and IAF. Importantly, under the assumption that the latent variables are free of measurement error, the relationships between latent variables estimated in a SEM are unbiased estimates of true relations among the constructs under study. Structural models are evaluated in terms of their 'model fit', that is, how well a model captures the structure inherent in the data. Conventionally, models are assumed to show good fit if the ratio $\chi^{2} / \mathrm{df}$ is less than 2 , the comparative fit index is larger than .95 (CFI; Bentler, 1990), and root-mean-square error of approximation (RMSEA) is smaller than .05 (Bentler, 1990; Browne and Cudeck, 1993). Significance and equivalence of specific parameters in the model are tested by constraining these parameters to their values under the null hypothesis, for example, by fixing the relationship between two latent variables (IAF and $g$ ) at zero, and by testing whether the resulting change in $\chi^{2}\left(\Delta \chi^{2}\right)$ is significant, with the degrees of freedom equal to the number of constrained parameters (cf. Bollen, 1989; Kline, 2005; see also Lövdén et al., 2013; Raz et al., 2005).

For CFA and SEM analyses IAF values were $z$-standardized within each age group. Models for the younger and older age group were fitted simultaneously with full information maximum likelihood estimation (FIML). By implementing estimation techniques originally developed in the context of pedigree analysis (Lange et al., 1976), FIML SEM provides the individual $-2 \log$ likelihood contributions to overall misfit. Hence, individuals with missing data can be included in the model without imputing missing data. In the present case, inclusion of the full COGITO sample allowed to establish a solid measurement model for arriving at a hierarchical model of intellectual abilities. Thereafter, we projected the IAF data from the EEG sub-sample $(n=85)$ into the latent space of intellectual abilities. A sound estimation of the correlation between intelligence factors and IAF would not have been possible on the latent level with the EEG sub-sample only, due to small sample size. Given that the EEG sub-samples were representative of the parent sample (with the exception of Spot-a-Word, older adults; see above), the parameter estimates reported in this article are unlikely to be biased. All raw scores were $z$-standardized before estimation. Therefore, the intercepts for the indicator variables and latent factors were constrained to zero to reduce the number of estimated parameters. To assure metric invariance of the measurement model of younger and older adults, the factor loadings for the indicator variables and the latent factors were constrained to be equal across age groups, as were the loadings for the first-order latent factors and the second-order factor $g$. Tests of measurement invariance are reported in the Results section. Confidence interval $(\mathrm{CI})$ boundaries for the relationship between IAF and $g$ are represented by those correlation coefficients where the model fit decreased by $\Delta \chi^{2}(1)=3.65$, which takes into account the skewed distribution of correlation coefficients and is a more conservative estimate for the $\mathrm{CI}$.

\section{Results}

Correlations between and within aggregated IAF and cognitive scores

As shown in Table 2, correlations between the parcels of the BIS constructs were homogeneous and medium to high within constructs [YA: $r \mathrm{~s}=.54-.68$; OA: $r \mathrm{~s}=.37-.69$; all Ps $<.01$ ], with smaller but significant correlations across constructs [YA: $r \mathrm{~s}=.22-.48$; OA: $r s=.18-.48$; all $P \mathrm{~s}<.01$, except $r=.18, P<.05]$. Furthermore, a highly significant and substantial correlation between IAF with eyes closed and eyes open was observed for both age groups (Table 3). Correlations between IAF and aggregated cognitive scores were all found to be positive but of small to medium size, with younger adults exhibiting a tendency towards higher correlations (Table 3). For the younger adults significant correlations were found between IAF measures and aggregated speed and memory scores, whereas for older adults only a significant correlation between IAF and the memory score could be observed. The correlation between mean IAF across the eyes closed and eyes open conditions and an overall composite score across the three ability measures was found to be $.38[P<.01]$ for the younger and $.19[P>.05]$ for the older adults. Overall, the correlation coefficients did not differ significantly between the two age groups [all $z \mathrm{~s}<1.16$; Ps $>.05$ ]. To estimate the association between mean IAF and the overall composite BIS score, we conducted a regression analysis with consecutively entering $\beta_{1}, \beta_{2}$, and $\beta_{3}$ into the following model: overall composite BIS score $=$ constant $+\beta_{1} \times$ age $+\beta_{2} \times$ mean $\mathrm{IAF}+\beta_{3} \times($ age $\times$ mean IAF). IAF explained a significant amount of variance of the overall composite BIS scores beyond age $\left[\beta_{1}: F(1,83)=84.55, P<.01 ; \beta_{2}\right.$ : $\left.F_{\text {change }}(1,82)=7.67, P<.01\right]$, but no significant interaction between IAF and age was found $\left[\beta_{3}: F_{\text {change }}(1,81)=0.87, P>.05\right]$. Taken togeth$\mathrm{er}$, this indicates that the association between IAF and intelligence did not differ significantly across our age groups; the partial correlation between IAF and $g$ after controlling for age was $.29(P<.01$; see Fig. 1$)$.

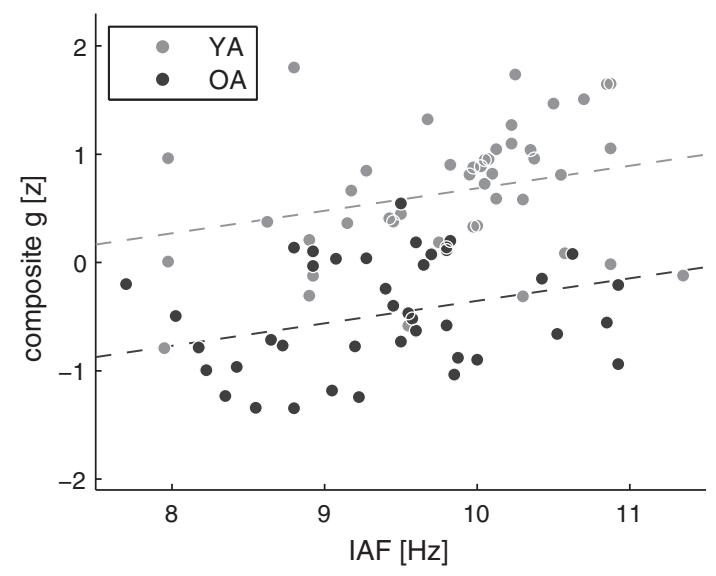

Fig. 1. Relationship between individual alpha frequency (IAF; mean across eyes closed and eyes open conditions) and z-standardized composite cognitive performance score across age groups across the BIS cognitive ability scores. Dashed lines indicate the slope of the relationship between IAF and composite cognitive performance. YA = younger adults; $\mathrm{OA}=$ older adults; $\mathrm{IAF}=$ individual alpha frequency. 
Table 4

Standardized factor loadings in the g-factor model.

\begin{tabular}{lllll}
\hline & \multicolumn{2}{l}{ Younger adults } & \multicolumn{2}{l}{ Older adults } \\
\hline g: speed & .84 & {$[.72-.96]$} & .79 & {$[.65-.93]$} \\
g: memory & .68 & {$[.55-.81]$} & .76 & {$[.62-.89]$} \\
g: reasoning & .73 & {$[.59-.87]$} & .78 & {$[.65-.90]$} \\
Speed: $\mathrm{spd}_{1}$ & .85 & {$[.78-.91]$} & .83 & {$[.76-.90]$} \\
Speed: $\mathrm{spd}_{2}$ & .80 & {$[.72-.87]$} & .77 & {$[.69-.84]$} \\
Speed: $\mathrm{spd}_{3}$ & .76 & {$[.68-.84]$} & .81 & {$[.74-.88]$} \\
Memory: mem $_{1}$ & .85 & {$[.77-.92]$} & .81 & {$[.72-.90]$} \\
Memory: mem $_{2}$ & .75 & {$[.67-.84]$} & .71 & {$[.61-.80]$} \\
Memory: mem $_{3}$ & .69 & {$[.59-.79]$} & .60 & {$[.50-.69]$} \\
Reasoning: $\mathrm{rsn}_{1}$ & .84 & {$[.76-.92]$} & .71 & {$[.62-.80]$} \\
Reasoning: $\mathrm{rsn}_{2}$ & .80 & {$[.72-.87]$} & .74 & {$[.64-.84]$} \\
Reasoning: $\mathrm{rsn}_{3}$ & .73 & {$[.64-.81]$} & .70 & {$[.60-.78]$} \\
\hline
\end{tabular}

Note. CFA second-order factor $(g)$ model for the full COGITO sample (YA: $n=145$; OA: $n=142$ ). Unstandardized factor loadings were constrained to be equal across age groups. $\mathrm{spd}_{\mathrm{i}}=$ perceptual speed parcels, mem $_{\mathrm{i}}=$ memory parcels, $\mathrm{rsn}_{\mathrm{i}}=$ reasoning parcels.

\section{Correlation between IAF and $g$}

To examine the factor structure of the BIS test, a CFA with the full COGITO sample data was conducted, with the three first-order latent factors of perceptual speed, memory and reasoning, and a second-order latent factor $g$. The unrestricted model fitted the data well $\left[\chi^{2}(66)=68.782\right.$ (YA: $\chi^{2}=47.02$; OA: $\chi^{2}=21.76$ ), RMSEA $=.017$, $\mathrm{CFI}=.997$, SRMR $=.040$ ], as did the model when specifying measurement invariance by constraining factor loadings to be equal across age groups [see Table $4 ; \chi^{2}(74)=84.28$ (YA: $\chi^{2}=53.43$; OA: $\left.\chi^{2}=30.84\right)$, RMSEA $=.038, \mathrm{CFI}=.990$, SRMR $\left.=.056\right]$. Specification of metric invariance of the measurement models for younger and older adults did not significantly reduce the fit of the model $\left[\Delta \chi^{2}(8)=15.49\right.$, $P>$.05]. Second-order standardized factor loadings on $g$ resulted in
$.84 / .79$ (YA/OA) for perceptual speed, .68/.76 for memory and .73/.78 for reasoning [all $P \mathrm{~s}<.001$ ]. The results are consistent with the assumption that a considerable amount of variance in the three cognitive abilities reflects individual differences in $g$. Standardized factor loadings for the first-order latent factors ranged between .60 and .85 [all Ps < .001; see Table 4].

Next, we projected the IAF latent factor, defined by the variables, IAF with eyes closed (EC) and IAF with eyes open (EO), into intellectual ability space established for the full COGITO sample by correlating it with $g$ (see Fig. 2). This model fitted the data well $\left[\chi^{2}(112)=121.81\right.$ (YA: $\chi^{2}=70.77 ; \quad$ OA: $\chi^{2}=51.04$ ), RMSEA $=.025, \quad$ CFI $=.991$, SRMR $=.076]$. Specification of metric invariance of the measurement model for IAF of younger and older adults did not significantly change the fit of the model $\left[\Delta \chi^{2}(1)=0.203, P>.05\right]$. The point estimate for the correlation between IAF and $g$ was $r=.40$ [confidence interval $(\mathrm{CI})=.14-.62]$. Fixing the covariance between IAF and $g$ to be zero led to significant reduction in model fit $\left[\chi^{2}(113)=130.05\right.$; $\left.\Delta \chi^{2}(1)=8.34, P<.01\right]$. Thus, we established a reliable and substantial correlation between IAF and $g$ for both age groups.

Estimating the correlation between IAF and $g$ freely for both age groups did not change the model fit significantly $\left[\chi^{2}(111)=121.30\right.$; $\left.\Delta \chi^{2}(1)=0.51, P>.05\right]$. When IAF-g correlations were estimated separately, the correlation was estimated at $.47[\mathrm{CI}=.14-.72]$ for the younger sample, and at .29 [CI $=-.12-.64]$ for the older sample. The non-significant IAF- $g$ correlation in the older sample as well as the lack of significant age group differences in correlations are likely to reflect a lack of statistical power associated with the small sample underlying the IAF measurement model.

After establishing a substantial correlation between IAF and intelligence at the level of second-order $g$, we examined whether there was any evidence within our data for specific associations between IAF and first-order factors of perceptual speed, memory, or reasoning. Importantly, estimating residual associations between the IAF latent

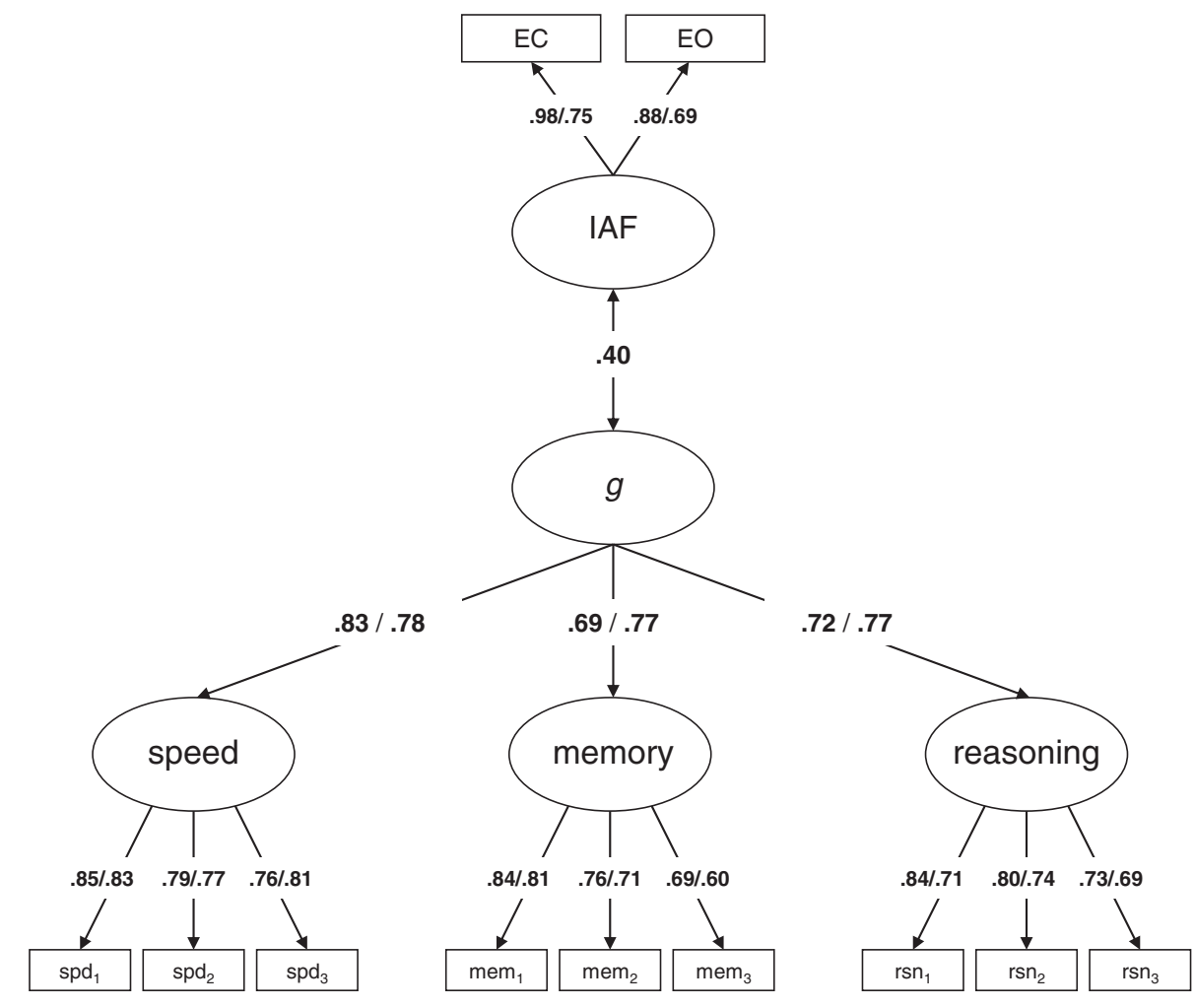

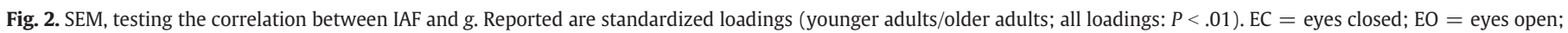

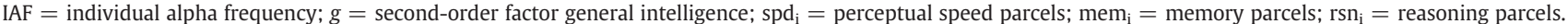


factor and each of the three first-order latent factors in the presence of the correlation between IAF and $g$ did not indicate any significant correlation between IAF and cognitive abilities beyond the correlation between IAF and $g$. None of the three introduced residual correlations significantly improved the fit of the model [IAF-speed: $\Delta \chi^{2}(1)=$ 0.62; IAF-memory: $\Delta \chi^{2}(1)=3.62$; IAF-reasoning: $\Delta \chi^{2}(1)=1.10$; all PS $>$.05]. Taken together, the correlation between IAF and $g$ substantially captures the covariation between IAF and reasoning, memory, and perceptual speed and our results are consistent with the hypothesis that IAF is associated to intelligence in a general, rather than specific, manner.

\section{Discussion}

Using structural equation modeling, we tested the hypothesis that individual alpha frequency (IAF) is related to cognitive performance at the level of the general factor of intelligence, or $g$. This hypothesis was based on the observation that IAF has been found to correlate with a broad range and variety of cognitive tasks (see Angelakis et al., 2004a, 2004b; Anokhin and Vogel, 1996; Clark et al., 2004; Giannitrapani, 1985; Klimesch et al., 1990, 1993, 1996; Lebedev, 1994; Mundy-Castle, 1958; Mundy-Castle and Nelson, 1960; Saletu and Grunberger, 1985; Surwillo, 1961, 1963, 1964). As predicted, we found a significant and substantial correlation $(r=.40)$ between IAF and $g$ that did not differ reliably between samples of younger and older adults. It is worth noting that a link between IAF and cognitive performance at the level of $g$, as modeled in this study, provides a parsimonious explanation for the diffuse pattern of correlations between IAF and cognitive performance that is found in the literature.

Our hypothesis of a substantial association between IAF and $g$ was informed by large-scale theories of neural organization that emphasize the functional significance of oscillatory activity (David and Friston, 2003; Jensen and Mazaheri, 2010; Klimesch, 2012; Klimesch et al., 2007; Sotero et al., 2007; Valdes-Hernandez et al., 2010). From the perspective of these theories, IAF qualifies as a candidate marker variable of individual differences in general aspects of neural organization that behaviorally manifest themselves as 'general intelligence'. In the following, we will elaborate on this line of reasoning.

\section{Neural mechanisms contributing to the association between IAF and $g$}

Mounting evidence suggests that intelligence cannot be attributed to a single brain region. For example, the parieto-frontal integration theory (P-FIT) by Jung and Haier (2007) suggests that individual differences found in intelligence and reasoning are best predicted by variations in a distributed network comprising interactions among several brain regions. A critical aspect of distributed networks is efficient communication among its elements. From this perspective, individual differences in intelligence also depend on differences in the efficiency of communication between widely distributed brain regions (cf. Anokhin et al., 1999; Jausovec and Jausovec, 2000; Lee et al., 2012; Neubauer and Fink, 2009a,b; Thatcher et al., 2005). Oscillatory neural activity together with the notion of the 'small-world' architecture of neural systems (cf. Sporns et al., 2004; Yu et al., 2008) complement neuroanatomy-based concepts of the biological basis of individual differences in cognitive functioning and intelligence (cf. Deary et al., 2006; Penke et al., 2010).

Neural oscillations have been postulated as a core mechanism in orchestrating the activity of the central nervous system, bridging the gaps from single neurons to cell assemblies and cortical networks (Wang, 2010). Synchronization of oscillatory activity offers an efficient mechanism for temporal coordination of neural activity in distributed networks (cf. Buzsáki and Draguhn, 2004). While faster oscillations in the gamma range most likely reflect local processing (cf. von Stein et al., 2000), slower oscillations, for example, in the alpha range, are well suited for long-range information integration
(Hummel and Gerloff, 2005; Mima et al., 2001). This line of reasoning would be consistent with the 'small-world' network characteristics of neural systems (cf. Sporns et al., 2004; Yu et al., 2008) in the sense that well connected local neural networks ('fast oscillations') are supplemented by a small fraction of long-range connections ('slow oscillations') involved in the coordination of local and distant networks, effectively reducing the number of explicit connections needed between distant cell assemblies (Buzsáki et al., 2004).

Specifically, it has been proposed that alpha oscillations may contribute to higher-order integration of information and top-down control by providing temporal frames for neural interactions via the timing of functional inhibition (cf. Jensen and Mazaheri, 2010; Jensen et al., 2002; Jokisch and Jensen, 2007; Klimesch, 2012; Klimesch et al., 2007; Tuladhar et al., 2007). According to this notion, faster IAF allows for faster alternations between 'open' and 'closed' states for information transfer, which in turn permits more efficient integration of information across brain regions. From a functional point of view, this proposition would explain why IAF accounts for some part of the between-person variance in $g$.

This line of reasoning ultimately leads to the question which brain properties co-determine the IAF of a given brain. According to neural mass models (cf. Jansen and Rit, 1995; Lopes da Silva et al., 1974), three global features of neuronal networks influence the frequency of oscillations: (a) local connectivity strength, that is, the number of synaptic contacts; (b) membrane time constants; and (c) propagation delays between distant cortical areas (David and Friston, 2003; Sotero et al., 2007). In line with the latter, a recent study demonstrated that white matter density is correlated with IAF in several distinct fiber tracts (Valdes-Hernandez et al., 2010). Thus, faster IAF, probably resulting from shorter propagation delays as a consequence of higher white matter density, would indeed be an expression of faster information transfer and synchronization between distant cortical areas. This is also in line with recent reports of an association of $g$ and white matter integrity (Deary et al., 2006; Penke et al., 2010).

\section{IAF and $g$ as trait markers}

As we have previously shown (Grandy et al., in press), IAF is an extraordinarily stable characteristic of individuals, even in the light of extensive cognitive training. This observation supports the idea that IAF is a neurophysiological trait marker, representing fundamental properties of individual brains that are not easily modifiable by experience. This notion would parallel the notion of $g$ as a highly stable person characteristic. Similar to $g$ (Deary et al., 2009; McGue et al., 1993), IAF exhibits high heritability (Lykken et al., 1974; Posthuma et al., 2001; Smit et al., 2006; van Baal et al., 2001; van Beijsterveldt and Boomsma, 1994; van Beijsterveldt and van Baal, 2002; Vogel, 1970), indicating that IAF is under strong genetic influence. Based on the preceding considerations, we consider it likely that a substantial portion of the variance shared between IAF and $g$ reflects individual differences that are genetic in origin.

\section{Age differences in the association between IAF and $g$ ?}

In this study, the correlation between IAF and $g$ did not differ reliably between younger and older adults. At the same time, when estimated for old adults alone, the correlation between IAF and $g$ observed did not differ reliably from zero. Statistical power may have been insufficient for detecting a reliable correlation between IAF and $g$ among older adults, and for picking up reliable age group differences in the association between IAF and $g$. Despite this inconclusive evidence, it is worthwhile to consider mechanisms that may lower IAF-g correlations with advancing adult age.

Groups of older adults tend to be more heterogeneous than groups of younger adults in relation to brain structure, chemistry, and function as well as behavior (Baltes et al., 2006; Lindenberger et al., 2013; 
Nyberg et al., 2012), presumably reflecting the accumulation of life experiences, genetic differences, the admixture of pathological conditions, and combinations thereof. It is conceivable that IAF may lose some of its association to general cognitive abilities across the life course, as other factors come into play. Research designs relying on the longitudinal assessment of multivariate changes within individuals are required to substantiate these speculations (Raz and Lindenberger, 2011).

\section{Summary and conclusions}

In this study, we made use of structural equation modeling to uncover an association between IAF and cognitive performance at the level of a second-order factor of general intelligence, or $g$. The observed correlation between IAF and $g$ offers a parsimonious explanation for previously reported IAF-cognition associations, which tended to escape explanations in terms of specific mechanisms or abilities. The present results support the hypothesis that individual differences in general intelligence are associated with individual differences in general oscillatory properties of the brain.

\section{Acknowledgments}

This work was supported by the Max Planck Society (including a grant from the Innovation Fund; M.FE.A.BILD0005), the Sofja Kovalevskaja Award (to ML) administered by the Alexander von Humboldt Foundation and donated by the German Federal Ministry for Education and Research (BMBF), the German Research Foundation (DFG; KFG 163), and the BMBF (CAI). UL was financially supported by the Gottfried Wilhelm Leibniz Award of the DFG. We thank Colin Bauer, Annette Brose, and all research assistants involved in data collection.

\section{Conflict of interest statement}

The authors have no conflict of interest to declare.

\section{References}

Adrian, E.D., Matthews, B.H.C., 1934. The Berger rhythm: potential changes from the occipital lobes in man. Brain 57, 355-385.

Adrian, E.D., Yamagiwa, D., 1935. The origin of the Berger rhythm. Brain 58, 323-351.

Angelakis, E., Lubar, J.F., Stathopoulou, S., 2004a. Electroencephalographic peak alpha frequency correlates of cognitive traits. Neurosci. Lett. 371, 60-63.

Angelakis, E., Lubar, J.F., Stathopoulou, S., Kounios, J., 2004b. Peak alpha frequency: an electroencephalographic measure of cognitive preparedness. Clin. Neurophysiol. $115,887-897$.

Anokhin, A., Vogel, F., 1996. EEG alpha rhythm frequency and intelligence in normal adults. Intelligence 23, 1-14

Anokhin, A.P., Lutzenberger, W., Birbaumer, N., 1999. Spatiotemporal organization of brain dynamics and intelligence: an EEG study in adolescents. Int. J. Psychophysiol. 33, 259-273.

Aurlien, H., Gjerde, I.O., Aarseth, J.H., Eldoen, G., Karlsen, B., Skeidsvoll, H., Gilhus, N.E., 2004. EEG background activity described by a large computerized database. Clin. Neurophysiol. 115, 665-673.

Baltes, P.B., Reuter-Lorenz, P.A., Rösler, F., 2006. Lifespan Development and the Brain: The Perspective of Biocultural Co-constructivism. Cambridge University Press, US, New York, NY.

Bell, A.J., Sejnowski, T.J., 1995. An information-maximization approach to blind separation and blind deconvolution. Neural Comput. 7, 1129-1159.

Bentler, P., 1990. Comparative fit indexes in structural models. Psychol. Bull. 107, $238-246$.

Berger, H., 1929. Über das Elektrenkephalogramm des Menschen. Eur. Arch. Psychiatry Clin. Neurosci. 87, 527-570.

Berger, H., 1930. Über das Elektrenkephalogramm des Menschen. J. Psychol. Neurol. 40, $160-179$.

Berger, H., 1933. Über das Elektrenkephalogramm des Menschen V. Eur. Arch. Psychiatry Clin. Neurosci. 98, 231-254.

Bollen, K.A., 1989. Structural Equations with Latent Variables. Wiley, New York, NY.

Bollen, K.A., 2002. Latent variables in psychology and the social sciences. Annu. Rev. Psychol. 53, 605-634.

Browne, M.W., Cudeck, R., 1993. Alternative was of assessing model fit. In: Bollen, K.A. Long, J.S. (Eds.), Testing Structural Equation Models. Sage Publications, Newbury Park, CA.
Buzsáki, G., Draguhn, A., 2004. Neuronal oscillations in cortical networks. Science 304, 1926-1929.

Buzsáki, G., Geisler, C., Henze, D.A., Wang, X.-J., 2004. Interneuron diversity series: circuit complexity and axon wiring economy of cortical interneurons. Trends Neurosci. 27, 186-193.

Chiang, A.K., Rennie, C.J., Robinson, P.A., van Albada, S.J., Kerr, C.C., 2011. Age trends and sex differences of alpha rhythms including split alpha peaks. Clin. Neurophysiol. $122,1505-1517$.

Clark, C.R., Veltmeyer, M.D., Hamilton, R.J., Simms, E., Paul, R., Hermens, D., Gordon, E., 2004. Spontaneous alpha peak frequency predicts working memory performance across the age span. Int. J. Psychophysiol. 53, 1-9.

David, O., Friston, K.J., 2003. A neural mass model for MEG/EEG: coupling and neuronal dynamics. NeuroImage 20,1743-1755.

Deakin, J.F.W., Exley, K.A., 1979. Personality and male-female influences on the EEG alpha rhythm. Biol. Psychol. 8, 285-290.

Deary, I.J., Bastin, M.E., Pattie, A., Clayden, J.D., Whalley, L.J., Starr, J.M., Wardlaw, J.M., 2006. White matter integrity and cognition in childhood and old age. Neurology $66,505-512$.

Deary, I.J., Johnson, W., Houlihan, L.M., 2009. Genetic foundations of human intelligence. Hum. Genet. 126, 215-232.

Deary, I.J., Penke, L., Johnson, W., 2010. The neuroscience of human intelligence differences. Nat. Rev. Neurosci. 11, 201-211.

Delorme, A., Makeig, S., 2004. EEGLAB: an open source toolbox for analysis of single-trial EEG dynamics including independent component analysis. J. Neurosci. Methods $134,9-21$.

Doppelmayr, M., Klimesch, W., Pachinger, T., Ripper, B., 1998. Individual differences in brain dynamics: important implications for the calculation of event-related band power. Biol. Cybern. 79, 49-57.

Ellingson, R.J., 1966. Relationship between EEG and test intelligence: a commentary. Psychol. Bull. 65, 91-98

Folstein, M.F., Folstein, S.E., McHugh, P.R., 1975. Mini-mental state. A practical method for grading the cognitive state of patients for the clinician. J. Psychiatr. Res. 12, 189-198.

Gasser, T., Bächer, P., Steinberg, H., 1985. Test-retest reliability of spectral parameters of the EEG. Electroencephalogr. Clin. Neurophysiol. 60, 312-319.

Giannitrapani, D., 1985. The Electrophysiology of Intellectual Functions. Karger, Basel. Grandy, T.H. Werkle-Bergner, M. Chicherio, C. Schmiedek, F., Lövdén, M., Lindenberger, U., 2013. Peak individual alpha frequency qualifies as a stable neurophysiological trait marker in healthy younger and older adults. Psychophysiology (in press).

Hummel, F., Gerloff, C., 2005. Larger interregional synchrony is associated with greater behavioral success in a complex sensory integration task in humans. Cereb. Cortex $15,670-678$

Jäger, A.O., Süß, H.M., Beauducel, A., 1997. Berliner Intelligenzstruktur-Test, BIS-TEST: Form 4. Handanweisung [The Berlin Intelligence Structure Test, BIS Test: Form 4. Test Manual] Hogrefe, Göttingen, Germany.

Jansen, B.H., Rit, V.G., 1995. Electroencephalogram and visual evoked potential generation in a mathematical model of coupled cortical columns. Biol. Cybern. 73, 357-366.

Jausovec, N., Jausovec, K., 2000. Differences in resting EEG related to ability. Brain Topogr. 12, 229-240

Jensen, O., Mazaheri, A., 2010. Shaping functional architecture by oscillatory alpha activity: gating by inhibition. Front. Hum. Neurosci. 4, 186.

Jensen, O., Gelfand, J., Kounios, J., Lisman, J.E., 2002. Oscillations in the alpha band $(9-12 \mathrm{~Hz})$ increase with memory load during retention in a short-term memory task. Cereb. Cortex 12, 877-882.

Johnson, W., Bouchard Jr., T.J., Krueger, R.F., McGue, M., Gottesman, I.I., 2004. Just one g: consistent results from three test batteries. Intelligence 32, 95-107.

Johnson, W., Nijenhuis, J.T., Bouchard Jr., T.J., 2008. Still just 1 g: consistent results from five test batteries. Intelligence 36, 81-95.

Jokisch, D., Jensen, O., 2007. Modulation of gamma and alpha activity during a working memory task engaging the dorsal or ventral stream. J. Neurosci. 27, 3244-3251.

Jung, R.E., Haier, R.J., 2007. The parieto-frontal integration theory (P-FIT) of intelligence: converging neuroimaging evidence. Behav. Brain Sci. 30, 135-154 (discussion 154-187)

Kanai, R., Rees, G., 2011. The structural basis of inter-individual differences in human behaviour and cognition. Nat. Rev. Neurosci. 12, 231-242.

Klimesch, W., 1996. Memory processes, brain oscillations and EEG synchronization. Int. J. Psychophysiol. 24, 61-100.

Klimesch, W., 1997. EEG-alpha rhythms and memory processes. Int. J. Psychophysiol. $26,319-340$.

Klimesch, W., 2012. Alpha-band oscillations, attention, and controlled access to stored information. Trends Cogn. Sci. 16, 606-617.

Klimesch, W., Schimke, H., Ladurner, G., Pfurtscheller, G., 1990. Alpha frequency and memory performance. J. Psychophysiol. 4, 381-390.

Klimesch, W., Schimke, H., Pfurtscheller, G., 1993. Alpha frequency, cognitive load and memory performance. Brain Topogr. 5, 241-251.

Klimesch, W., Doppelmayr, M., Schimke, H., Pachinger, T., 1996. Alpha frequency, reaction time, and the speed of processing information. J. Clin. Neurophysiol. 13, 511-518.

Klimesch, W., Sauseng, P., Hanslmayr, S., 2007. EEG alpha oscillations: the inhibitiontiming hypothesis. Brain Res. Rev. 53, 63-88.

Kline, R.B., 2005. Principles and Practice of Structural Equation Modeling, 2nd ed. Guilford Press, New York, NY.

Kondacs, A., Szabó, M., 1999. Long-term intra-individual variability of the background EEG in normals. Clin. Neurophysiol. 110, 1708-1716.

Lange, K., Westlake, J., Spence, M.A., 1976. Extensions to pedigree analysis. III. Variance components by the scoring method. Ann. Hum. Genet. 39, 485-491.

Lebedev, A.N., 1994. The neurophysiological parameters of human memory. Neurosci. Behav. Physiol. 24, 254-259. 
Lee, T.-W., Wu, Y.-T., Yu, Y.W., Wu, H.-C., Chen, T.-J., 2012. A smarter brain is associated with stronger neural interaction in healthy young females: a resting EEG coherence study. Intelligence 40, 38-48.

Lindenberger, U., Burzynska, A.Z., Nagel, I.E., 2013. Heterogeneity in frontal lobe aging, In: Stuss, D.T., Knight, R.T. (Eds.), Principles of Frontal Lobe Functions, 2nd ed. Oxford University Press, New York, pp. 609-627.

Lopes da Silva, F.H., Hoeks, A., Smits, H., Zetterberg, L.H., 1974. Model of brain rhythmic activity. The alpha-rhythm of the thalamus. Kybernetik 15, 27-37.

Lövdén, M., Laukka, E.J., Rieckmann, A., Kalpouzos, G., Li, T.-Q., Jonsson, T., Wahlund, L.-O., Fratiglioni, L., Bäckman, L., 2013. The dimensionality of between-person differences in white matter microstructure in old age. Hum. Brain Mapp. 34, 1386-1398.

Lykken, D.T., Tellegen, A., Thorkelson, K., 1974. Genetic determination of EEG frequency spectra. Biol. Psychol. 1, 245-259.

McGue, M., Bouchard Jr., T.J., Iacono, W.G., Lykken, D.T., McClearn, R.P.G.E., 1993. Behavioral Genetics of Cognitive Ability: A Life-span Perspective. Nature, Nurture \& Psychology.American Psychological Association, Washington, DC, US 59-76.

Mima, T., Oluwatimilehin, T., Hiraoka, T., Hallett, M., 2001. Transient interhemispheric neuronal synchrony correlates with object recognition. J. Neurosci. 21, 3942-3948.

Mundy-Castle, A.C., 1958. Electrophysiological correlates of intelligence. J. Pers. 26, 184-199.

Mundy-Castle, A.C., Nelson, G.K., 1960. Intelligence, personality and brain rhythms in a socially isolated community. Nature $185,484-485$.

Muthén, L.K., Muthén, B.O., 1998-2010. Mplus User's Guide, 6th ed. Muthén \& Muthén, Los Angeles, CA.

Narr, K.L., Woods, R.P., Thompson, P.M., Szeszko, P., Robinson, D., Dimtcheva, T., Gurbani, M., Toga, A.W., Bilder, R.M., 2007. Relationships between IQ and regional cortical gray matter thickness in healthy adults. Cereb. Cortex 17, 2163-2171.

Neubauer, A.C., Fink, A., 2009a. Intelligence and neural efficiency. Neurosci. Biobehav. Rev. 33, 1004-1023.

Neubauer, A.C., Fink, A. 2009b. Intelligence and neural efficiency: measures of brain activation versus measures of functional connectivity in the brain. Intelligence 37, 223-229.

Niedermeyer, E., Lopes da Silva, F.H. (Eds.), 1999. Electroencephalography: Basic Principles, Clinical Applications, and Related Fields, 2nd ed. Lippincott Williams \& Wilkins, Philadelphia.

Nyberg, L., Lövdén, M., Riklund, K., Lindenberger, U., Bäckman, L., 2012. Memory aging and brain maintenance. Trends Cogn. Sci. 16, 292-305.

Oostenveld, R., Praamstra, P., 2001. The five percent electrode system for highresolution EEG and ERP measurements. Clin. Neurophysiol. 112, 713-719.

Oostenveld, R., Fries, P., Maris, E., Schoffelen, J.M., 2011. FieldTrip: open source software for advanced analysis of MEG, EEG, and invasive electrophysiological data. Comput. Intell. Neurosci. 2011, 156869.

Penke, L., Munoz Maniega, S., Murray, C., Gow, A.J., Hernandez, M.C., Clayden, J.D., Starr, J.M., Wardlaw, J.M., Bastin, M.E., Deary, I.J., 2010. A general factor of brain white matter integrity predicts information processing speed in healthy older people. J. Neurosci. 30, 7569-7574.

Posthuma, D., Neale, M., Boomsma, D., de Geus, E., 2001. Are smarter brains running faster? Heritability of alpha peak frequency, IQ and their interrelation. Behav. Genet. 31, 567-579.

Raz, N., Lindenberger, U., 2011. Only time will tell: cross-sectional studies offer no solution to the age-brain-cognition triangle: comment on Salthouse (2011). Psychol. Bull. 137, 790-795.

Raz, N., Lindenberger, U., Rodrigue, K.M., Kennedy, K.M., Head, D., Williamson, A., Dahle, C., Gerstorf, D., Acker, J.D., 2005. Regional brain changes in aging healthy adults: general trends, individual differences and modifiers. Cereb. Cortex 15, 1676-1689.

Saletu, B., Grunberger, J., 1985. Memory dysfunction and vigilance: neurophysiological and psychopharmacological aspects. Ann. N. Y. Acad. Sci. 444, 406-427.

Salinsky, M.C., Oken, B.S., Morehead, L., 1991. Test-retest reliability in EEG frequency analysis. Electroencephalogr. Clin. Neurophysiol. 79, 382-392.
Schmiedek, F., Hildebrandt, A., Lövdén, M., Wilhelm, O., Lindenberger, U., 2009. Complex span versus updating tasks of working memory: the gap is not that deep. J. Exp. Psychol. Learn. 35, 1089-1096.

Schmiedek, F., Lövdén, M., Lindenberger, U., 2010. Hundred days of cognitive training enhance broad cognitive abilities in adulthood: findings from the COGITO study. Front. Aging Neurosci. 2, 27 (pii).

Smit, C.M., Wright, M.J., Hansell, N.K., Geffen, G.M., Martin, N.G., 2006. Genetic variation of individual alpha frequency (IAF) and alpha power in a large adolescent twin sample. Int. J. Psychophysiol. 61, 235-243.

Sotero, R.C., Trujillo-Barreto, N.J., Iturria-Medina, Y., Carbonell, F., Jimenez, J.C., 2007. Realistically coupled neural mass models can generate EEG rhythms. Neural Comput. 19, 478-512.

Spearman, C., 1904. "General intelligence," objectively determined and measured. Am. J. Psychol. 15, 201-292.

Sporns, O., Chialvo, D.R., Kaiser, M., Hilgetag, C.C., 2004. Organization, development and function of complex brain networks. Trends Cogn. Sci. 8, 418-425.

Surwillo, W.W., 1961. Frequency of the 'alpha' rhythm, reaction time and age. Nature $191,823-824$

Surwillo, W.W., 1963. The relation of simple response time to brain-wave frequency and the effects of age. Electroencephalogr. Clin. Neurophysiol. 15, 105-114.

Surwillo, W.W., 1964. The relation of decision time to brain wave frequency and to age Electroencephalogr. Clin. Neurophysiol. 16, 510-514.

Thatcher, R.W., North, D., Biver, C., 2005. EEG and intelligence: relations between EEC coherence, EEG phase delay and power. Clin. Neurophysiol. 116, 2129-2141.

Toga, A.W., Thompson, P.M., 2005. Genetics of brain structure and intelligence. Annu. Rev. Neurosci. 28, 1-23.

Tuladhar, A.M., ter Huurne, N., Schoffelen, J.M., Maris, E., Oostenveld, R., Jensen, O. 2007. Parieto-occipital sources account for the increase in alpha activity with working memory load. Hum. Brain Mapp. 28, 785-792.

Valdes-Hernandez, P.A., Ojeda-Gonzalez, A., Martinez-Montes, E., Lage-Castellanos, A. Virues-Alba, T., Valdes-Urrutia, L., Valdes-Sosa, P.A., Valdes-Hernandez, P.A., Ojeda-Gonzalez, A., Martinez-Montes, E., Lage-Castellanos, A., Virues-Alba, T., Valdes-Urrutia, L., Valdes-Sosa, P.A., 2010. White matter architecture rather than cortical surface area correlates with the EEG alpha rhythm. Neurolmage 49, 2328-2339.

van Baal, G., van Beijsterveldt, C., Molenaar, P., Boomsma, D., de Geus, E., 2001. A genetic perspective on the developing brain: electrophysiological indices of neural functioning in young and adolescent twins. Eur. Psychol. 6, 254-263.

van Beijsterveldt, C.E.M., Boomsma, D.I., 1994. Genetics of the human electroencephalogram (EEG) and event-related brain potentials (ERPs): a review. Hum. Genet. 94, 319-330.

van Beijsterveldt, C.E.M., van Baal, G., 2002. Twin and family studies of the human electroencephalogram: a review and a meta-analysis. Biol. Psychol. 61, 111-138.

Vogel, F., 1970. The genetic basis of the normal human electroencephalogram (EEG). Humangenetik 10, 91-114.

Vogel, W., Broverman, D.M., 1964. Relationship between EEG and test intelligence: a critical review. Psychol. Bull. 62, 132-144.

von Stein, A., Chiang, C., Konig, P., 2000. Top-down processing mediated by interarea synchronization. Proc. Natl. Acad. Sci. U. S. A. 97, 14748-14753.

Wang, X.J., 2010. Neurophysiological and computational principles of cortical rhythms in cognition. Physiol. Rev. 90, 1195-1268.

Wilhelm, O., Schulze, R., 2002. The relation of speeded and unspeeded reasoning with mental speed. Intelligence 30, 537-554.

Yu, S., Huang, D., Singer, W., Nikolic, D., 2008. A small world of neuronal synchrony. Cereb. Cortex 18, 2891-2901.

Ziegler, G., Dahnke, R., Gaser, C., 2012. Models of the aging brain structure and individual decline. Front. Neuroinformatics 6. 